\title{
Interval versus Continuous Training
}

\section{Spanoudaki S*}

Department of Sports Medicine \& Biology of PhysicalActivity, Faculty of Physical Education and Sports Science, National \& Kapodistrian University ofAthens, Greece

Continuous and interval training are widely used in sports practice in order to improve performance in athletes, or to promote health in sedentary populations. Moreover, both types of exercise are used in rehabilitation programs of patients with chronic diseases [1,2]. Interval training is considered as 'better training' and consists of repeated periods of high intensity exercise alternating with periods of lower intensity, whereas continuous exercise is characterized by constant submaximal workload. During the 1930's interval training became popular in track and field events (Woldermar Geschler, a German running coach first introduced this type of exercise). While in the early 1960's the Studies of Astrand et al. [3] and Christensen et al. [4] opened a new field in exercise physiology and training. Afterwards, few studies examined the cardiovascular and metabolic responses between the two types with contradictory results [5-8]. The differences between the studies are attributed to the variability of the exercise protocol (duration, type, and intensity, ratio of work at high intensity/low or complete rest), which in turn results in recruitment of different energy systems.

The preference in interval training compared to constant is attributed to the fact that high intensity exercise can be sustained longer compared to constant, imposing greater stress to the body. As a consequence, there is greater functional improvement in both the muscle and cardiorespiratory system. Daussin et al. $[9,10]$, found that interval training in sedentary subjects, improved both central and peripheral components of oxygen consumption $\left(\mathrm{VO}_{2 \max }\right)$, while continuous training was associated with greater $\mathrm{O}_{2}$ extraction. Interval training, is also beneficial for highly trained athletes, who difficult enhance further endurance performance. Studies show that such athletes can improve endurance performance mainly through high intensity interval training. The alternations between periods of very high intensity (greater that anaerobic threshold) and brief periods of low intensity/ or inactivity, allow a partial recovery, which results in repeatedly stress of physiological systems to a greater extent than that required during exercise $[11,12]$, resulting greater adaptations. Furthermore, Interval exercise seemed to be preferable in patients with cardiac problems compared to continuous. This is because, it challenges the heart's pumping ability, which in turn causes cardiac remodeling [13].

Both continuous and interval training are beneficial for athletes and sport participants, either by increasing performance or promoting health and well being. More studies need to be conducted in order to compare the two types of training. Also, we should consider that both types are essential, when designing an exercise program. The age, the physical condition of the participant, the training phase (periodization), the targeting energy system and of course the goal of the program, determine which type of exercise is to be chosen.

\section{References}

1. Puhan MA, Schunemann HJ, Frey M, Schrplatz M, Bachmann LM (2005) How should COPD patients exercise during respiratory rehabilitation? Comparison of exercise modalities and intensities to treat skeletal muscle dysfunction. Thorax 60: 367-375.

2. Samitz G, Bachl N (1991) Physical training programs and their effects on aerobic capacity and coronary risk profile in sedentary individuals. Design of a long-term exercise training program. J Sports Med Phys Fitness 31: 283-293.

3. Astrand I, Astrand PO, Christensen EH, Hedman R (1960) Intermittent Muscular Work. Acta Physiol Scand 48: 448-453.

4. Christensen EH, Hedman R, Saltin B (1960) Intermittent and Continuous Running. Acta Physiol Scand 50: 269-286.

5. Essen B, Hagenfeldt L, Kaijser L (1977) Utilization of blood-borne and intramuscular substrates during continuous and intermittent exercise in man. J Physiol 265: 489-506.

6. Essen B (1978) Glycogen depletion of different fibre types in human skeleta muscle during intermittent and continuous exercise. Acta Physiol Scand 103 446-455.

7. Christmass MA, Dawson B, Arthur PG (1999) Effect of work and recovery duration on skeletal muscle oxygenation and fuel use during sustained intermittent exercise. Eur J Appl Physiol Occup Physiol 80: 436-447.

8. Christmass MA, Dawson B, Passeretto P, Arthur PG (1999) A comparison of skeletal muscle oxygenation and fuel use in sustained continuous and intermittent exercise. Eur J Appl Physiol Occup Physiol 80: 423-435.

9. Daussin FN, Ponsot E, Dufor SP, Lonsdorfer-Wolf E, Doutreleau S, et al. (2007) Improvement of $\mathrm{VO}_{2 \max }$ by cardiac output and oxygen extraction adaptation during intermittent versus continuous endurance training. Eur J Appl Physio 101: $377-383$

10. Daussin FN, Zoll J, Dufour SP, Ponsot E, Lonsdorfer-Wolf E, et al. (2008) Effects of interval versus continuous training on cardiorespiratory and mitochondrial functions: relationship to aerobic performance improvements in sedentary subjects. Am J Physiol Regul Integr Comp Physiol 295: R264-R272.

11. Laursen PB, Jenkins DG (2002) The Scientific basis for high intensity interval training: optimizing training programmes and maximising performance in highly trained endurance athletes. Sports Med 32: 53-73.

12. Daniels J, Scardina N (1984) Interval training and performance. Sports Med 1: 327-334.

13. Wisloff U, Stoylen A, Loennechen JP, Bruvold M, Rognmo O, et al. (2007) Superior Cardiovascular Effect of Aerobic Interval Training versus Moderate Continuous Training in Heart Failure Patients: A randomized Study. Circulation 115: 3086-3094.

*Corresponding author: Spanoudaki S, Department of Sports Medicine \& Biology of Physical Activity, Faculty of Physical Education and Sports Science, National \& Kapodistrian University of Athens, 41 Ethnikis Antistasis, Dafni, Vrilissia 15235, Greece, Tel: 0030 - 2108103282; Fax: 0030 - 2109708367; E-mail: sspanoud@ yahoo.gr

Received September 12, 2011; Accepted November 19, 2011; Published November 23, 2011

Citation: Spanoudaki S (2011) Interval versus Continuous Training. J Sport Medic Doping Studie 1:e102. doi:10.4172/2161-0673.1000e102

Copyright: (C) 2011 Spanoudaki S. This is an open-access article distributed under the terms of the Creative Commons Attribution License, which permits unrestricted use, distribution, and reproduction in any medium, provided the original author and source are credited. 